\title{
OPTIMIZATION OF HEAT TRANSFER PROPERTIES OF NANOFLUID FLOW OVER A SHRINKING SURFACE THROUGH MATHEMATICAL MODELING
}

\author{
A. BHANDARI* and R.K. PAVAN KUMAR PANNALA \\ University of Petroleum and Energy Studies (UPES) \\ Department of Mathematics, School of Engineering \\ Energy Acres Building, Bidholi \\ Dehradun- 248007, Uttarakhand, INDIA \\ E-mail: pankaj.anupam6@gmail.com
}

\begin{abstract}
In the current study, a three dimensional incompressible magnetohydrodynamic (MHD) nanofluid flow over a shrinking surface with associated thermal buoyancy, thermal radiation, and heating absorption effects, as well as viscous dissipation have been investigated. The model has been represented in a set of partial differential equations and is transformed using suitable similarity transformations which are then solved by using the finite element method through COMSOL. The results for velocity and temperature profiles are provided for various values of the shrinking parameter, Biot's number, heat generation/absorption parameter, thermal Grashof number, nanoparticle volume fraction, permeability parameter, magnetic parameter and radiation parameter.
\end{abstract}

Key words: magnetohydrodynamics, nanofluid, velocity, 3D flow, thermal and heat absorption effects, shrinking surface.

\section{Introduction}

Magnetohydrodynamics (MHD) is concerned with the study of the interaction between magnetic fields and fluid conductors of electricity. The body force which arises in MHD is the Lorentz force that occurs when an electric current flows at an angle to the direction of an applied magnetic field. In the beginning, the researchers were working on the basic structure of a magnetic fluid flowing over a boundary layer, but during the last few decades, numerous scientists motivated us to examine the magnetohydrodynamic stream of nanofluid flow over various geometries in order to examine heat transfer properties and possible industrial applications.

The magnetohydrodynamic mixed convection inside the center heater has been investigated numerically using the finite volume method [1]. Researchers have studied the flow of MHD over a shrinking surface in the presence of a magnetic field $[2,3]$. Various physical properties of MHD flow over a stretching sheet have been investigated $[4,5]$. The stagnation-point flow of a nanofluid over a vertical permeable surface has been studied numerically using the Runge-Kutta method [6]. The heat mass transfer on MHD nanofluid flow over a two-dimensional sheet in the presence of considered physical parameters have been examined [7-9]. In the presence of radiation and chemical reaction parameters, the heat and mass transfer of a Casson nanofluid flow through a vertical plate has been examined [10]. Heat and mass flux with isothermal properties of nanofluid flow have been investigated [11,12].

The heat and mass transfer analysis of an MHD nanofluid flow with homogeneous-heterogeneous reactions have been investigated and the size of the nanoparticles has also been analyzed [13-15]. The peristaltic flow of a nanofluid in a channel with compliant walls has been examined [16]. The Powell-Eyring nanofluid, Jeffery nanofluid, and second-grade nanofluid have been studied in different flow geometries for velocity, temperature and concentration distributions [17-19]. Important physical aspects of a nanofluid flow

\footnotetext{
* To whom correspondence should be addressed
} 
with convection have been explored [20,21]. The stagnation point flow of a nanofluid and its behavior in a porous medium have been studied with dimensional analysis $[22,23]$. The effects of variable thickness of the flow through a stretching sheet have been analyzed. The developed mathematical model has been solved using the natural decomposition method [24]. A numerical study has been presented for a nanofluid flow in a rotating channel containing a carbon nanotube [25]. A mathematical model has been developed for Darcy Forchheimer's effects on the three-dimensional nanofluid flow [26]. Radiation in a nanofluid flow through a semi-permeable duct with Joule heating has been investigated [27]. A nanofluid flow over a cone with bioconvection and entropy generation has been investigated [28]. A similarity transformation has been used to study the model for a nanofluid flow over a cone with solutal stratification [29].

Heat and mass transfer analysis for a Casson nanofluid flow over a rotating cylinder has been carried out [30]. An increase in the relaxation time increases the boundary layer width of nanofluid flow over a static wedge [31]. Nanofluid transportation due to an electric field has been studied numerically using the control volume method [32]. Heat transfer analysis for the rotational flow over a stretching surface has been examined [33]. The magnetohydrodynamic flow of a Maxwell fluid over an exponentially stretching surface has been studied with nanomaterials on the surface [34]. A numerical study has been carried out on the thin film flow of a nanofluid [35]. The results for a silver-water nanofluid flow over a stretching cylinder with homogeneous-heterogeneous reactions have been presented [36]. An unsteady flow of carbon nanotubes based nanofluid with homogeneous-heterogeneous reactions has been examined [37]. The silver water-based nanofluid flow over a nonlinear stretching cylinder has been studied numerically using the shooting method [38]. The magnetohydrodynamic couple stress nanofluid flow over an exponentially stretching surface with Joule heating and viscous dissipation effects have been studied [39].

The nanoparticle's solidification process in a heat storage system has been investigated through the finite element method [40]. The radiative heat transfer of a water-based nanofluid has been studied numerically through the control volume method [41]. A three-dimensional nanofluid flow over an exponentially stretching surface has been studied by the homotopy analysis method [42]. The viscoelastic flow of a non-Newtonian nanofluid has been studied through the finite difference method [43]. Heat transfer enhancement has been presented for a Maxwell nanofluid [44]. A micropolar nanofluid flow over a stretching sheet has been investigated with the help of the finite difference method [45]. The magnetohydrodynamic nanofluid flow on a stretching sheet has been investigated with slip conditions [46].

In the present study, a three-dimensional MHD nanofluid flow over a porous shrinking surface is considered in the presence of a magnetic field. The thermal and heat absorption effects along with thermal radiation and viscous dissipation have been presented in the flow. Using a similarity transformation, a set of governing partial differential equations has been transformed into nonlinear ordinary differential equations. The solution of nonlinear-coupled differential equations is achieved by the finite element method. COMSOL Multiphysics has been used to obtain the solution of the nonlinear coupled differential equations. The results for velocity and temperature distributions are obtained for different values of a shrinking parameter $(A)$, Biot's number $(\mathrm{Bi})$, heat generation/absorption parameter $(\chi)$, thermal Grashof number $(\mathrm{Gr})$, nanoparticle volume fraction $(\varphi)$, permeability parameter $(K p)$, magnetic parameter $(M)$ and radiation parameter $(R)$.

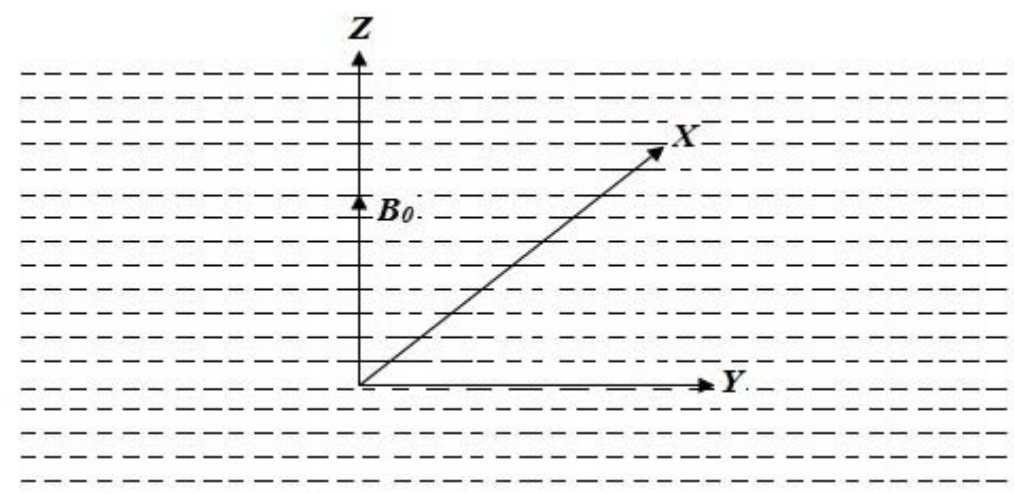

Fig.1. Schematic diagram of the flow. 


\section{Formulation of the problem}

A three-dimensional conducting nanofluid flow over a porous shrinking surface is considered as shown in Fig.1. The uniform magnetic field $\left(\boldsymbol{B}_{0}\right)$ is directed in the $z$ direction. The induced magnetic field is considered zero as compared to the applied magnetic field. The effect of the electric field is also considered zero. A mathematical model has been extended to study the thermal buoyancy and heat absorption effects along with the thermal radiation and heat dissipation. The change in the viscosity due to the applied magnetic field is also considered. The modified model for the proposed flow is as follows

$$
\begin{aligned}
& \frac{\partial u}{\partial x}+\frac{\partial v}{\partial y}+\frac{\partial u}{\partial z}=0 \\
& u \frac{\partial u}{\partial x}+v \frac{\partial u}{\partial y}+w \frac{\partial u}{\partial z}=v\left(1+\frac{3}{2} \varphi_{1} \lambda\right) \frac{\partial^{2} u}{\partial z^{2}}-\frac{\sigma B_{0}^{2}}{\rho_{n}} u-\frac{\psi}{K_{p}^{\prime}} u+g \beta\left(T-T_{\infty}\right), \\
& u \frac{\partial v}{\partial x}+v \frac{\partial v}{\partial y}+w \frac{\partial v}{\partial z}=v\left(1+\frac{3}{2} \varphi_{1} \lambda\right) \frac{\partial^{2} v}{\partial z^{2}}-\frac{\sigma B_{0}^{2}}{\rho_{n}} v-\frac{\psi}{K_{p}^{\prime}} v+g \beta\left(T-T_{\infty}\right) \\
& u \frac{\partial T}{\partial x}+v \frac{\partial T}{\partial y}+w \frac{\partial T}{\partial z}=\alpha_{n} \frac{\partial^{2} T}{\partial z^{2}}-\frac{1}{\left(\rho C_{p}\right)_{n}} \frac{\partial q_{r}}{\partial z}+\frac{\mu_{1}}{\left(\rho C_{p}\right)_{n}}\left(\frac{\partial w}{\partial z}\right)^{2}-\frac{Q_{0}}{\left(\rho C_{p}\right)_{n}}\left(T-T_{\infty}\right)
\end{aligned}
$$

The boundary conditions for the model are as follows

$$
\begin{aligned}
& u=a x, \quad v=a(m-1) y, \quad w=-W, \quad-k_{f} \frac{\partial T}{\partial z}=h\left(T_{f}-T\right) \quad \text { at } \quad z=0, \\
& u \rightarrow 0, \quad v \rightarrow 0, \quad T \rightarrow T_{\infty} \quad \text { as } \quad z \rightarrow \infty
\end{aligned}
$$

where $a$ is a constant and becomes positive or negative for a stretching or shrinking sheet, respectively. Along the $x$ direction, the considered shrinking sheet shrinks for $m=1$ and it shrinks axisymmetric for $m=2$.

In Eqs (2.2)-(2.3), the expression $g \beta\left(T-T_{\infty}\right)$ indicates the thermal buoyancy effects and the expression $\frac{Q_{0}}{\left(\rho C_{p}\right)_{n}}\left(T-T_{\infty}\right)$ represents the heat absorption effect. The expression $\left(1+\frac{3}{2} \varphi_{1} \lambda\right)$ indicates the magnetic field dependent viscosity. If we exclude the effects of thermal buoyancy, heat absorption effects and magnetic field dependent viscosity effects in the flow, this model will be reduced to the model previously published in [2].

The nanofluid characteristic parameters can be written as follows[24]

$$
\begin{aligned}
& \rho_{n}=(1-\varphi) \rho_{f}+\varphi \rho_{s}, \\
& \mu_{n}=\frac{\mu_{f}}{(1-\varphi)^{2.5}}, \\
& \alpha_{n}=\frac{k_{n}}{\left(\rho C_{p}\right)_{n}},
\end{aligned}
$$




$$
\begin{aligned}
& \left(\rho C_{p}\right)_{n}=(1-\varphi)\left(\rho C_{p}\right)_{f}+\varphi\left(\rho C_{p}\right)_{s}, \\
& \frac{k_{n}}{k_{f}}=\frac{k_{s}+2 k_{f}-2 \varphi\left(k_{f}-k_{s}\right)}{k_{s}+2 k_{f}+\varphi\left(k_{f}-k_{s}\right)} .
\end{aligned}
$$

The following similarity transformations can be written in the dimensionless form of the boundary layer equations

$$
\begin{aligned}
& u=c x \frac{d f}{d \xi}, \quad v=c(m-1) y \frac{d f}{d \xi}, \quad w=-\sqrt{c \frac{\mu_{f}}{\rho_{f}}} m f(\xi), \\
& \xi=\sqrt{\frac{c \rho_{f}}{\mu_{f}}} z, \quad \theta(\xi)=\frac{T-T_{\infty}}{T_{f}-T_{\infty}} .
\end{aligned}
$$

The radiative heat flux using the Rosseland approximation [25] is given as

$$
q_{r}=-\frac{16 \sigma^{\prime}}{3 k_{l}} T^{3} \frac{\partial T}{\partial z}
$$

Therefore, Eq.(2.4) takes the form

$$
u \frac{\partial T}{\partial x}+v \frac{\partial T}{\partial y}+w \frac{\partial T}{\partial z}=\alpha_{n} \frac{\partial^{2} T}{\partial z^{2}}-\frac{16 \sigma^{\prime} T_{\infty}^{3}}{3 k_{1}\left(\rho C_{p}\right)_{n}} \frac{\partial^{2} T}{\partial z^{2}}+\frac{\mu_{1}}{\left(\rho C_{p}\right)_{n}}\left(\frac{\partial w}{\partial z}\right)^{2}-\frac{Q_{0}}{\left(\rho C_{p}\right)_{n}}\left(T-T_{\infty}\right) .
$$

The dimensionless quantities are presented as follows

$$
\begin{aligned}
& M=\frac{\sigma B_{0}^{2}}{\left(C_{p}\right)_{f}}, \quad K_{p}=\frac{\mu_{f}}{\rho_{f} c K_{p}^{\prime}}, \quad R=\frac{16 \sigma^{\prime} T_{\infty}^{3}}{3 k_{f} k_{l}}, \quad E_{c}=\frac{\mu_{f}}{\left(T_{f}-T_{\infty}\right)\left(\rho C_{p}\right)_{f}}, \\
& \operatorname{Pr}=\frac{\mu_{f}\left(\rho C_{p}\right)_{f}}{\rho_{f} k_{f}}, \quad A=\frac{a}{c}, \quad k=\frac{k_{n}}{k_{f}}, \quad \mathrm{Gr}=\frac{\mu_{f} g \beta\left(T_{f}-T_{\infty}\right)}{\rho_{f}\left(\rho C_{p}\right)_{f}}, \quad \chi=\frac{Q_{0}}{\left(C_{p}\right)_{f}^{2}} .
\end{aligned}
$$

Using Eqs (2.6)-(2.11) and Eq.(2.14), Eqs (2.2), (2.3) and (2.13) can be transformed into nonlinear coupled ordinary differential equations as

$$
\begin{aligned}
& \left(1+\frac{3}{2} \varphi_{1} \lambda\right) \frac{d^{3} f}{d \xi^{3}}+(1-\varphi)^{2.5}\left[1-\varphi+\left(\frac{\rho_{s}}{\rho_{f}}\right) \varphi\left[m f \frac{d^{2} f}{d \xi^{2}}-\left(\frac{d f}{d \xi}\right)^{2}\right]+\right. \\
& -\left[(1-\varphi)^{2.5} M+K_{p}\right] \frac{d f}{d \xi}-\operatorname{Gr} \theta=0,
\end{aligned}
$$




$$
\frac{1}{\operatorname{Pr}}(k+R) \frac{d^{2} \theta}{d \xi^{2}}+\frac{\operatorname{Ec} m^{2}}{(1-\varphi)^{2.5}}\left(\frac{d f}{d \xi}\right)^{2}+\left[1-\varphi+\left\{\frac{\left(\rho C_{p}\right)_{s}}{\left(\rho C_{p}\right)_{f}}\right\} \varphi\right] m f \frac{d \theta}{d \xi}+\chi \theta=0
$$

The transformed boundary conditions are

$$
\begin{aligned}
& f=f_{w}, \quad \frac{d f}{d \xi}=A, \quad \frac{d \theta}{d \xi}=-B_{i}(1-\theta) \quad \text { at } \quad \xi=0, \\
& \frac{d f}{d \xi} \rightarrow 0, \quad \theta \rightarrow 0 \quad \text { as } \quad \xi \rightarrow \infty .
\end{aligned}
$$

Here, $\quad f_{w}=\frac{w}{m \sqrt{\left(c \frac{\mu_{f}}{\rho_{f}}\right)}}$ with $\quad f_{w}>0, \quad A=\frac{a}{c} \quad$ and $\quad B_{i}=\frac{h}{k_{f}} \sqrt{\frac{\mu_{f}}{c \rho_{f}}}$.

The physical quantities of practical interest are the skin friction coefficient and heat transfer rate which can be expressed as

$$
\begin{array}{ll}
C_{f}=-\frac{\tau_{w}}{\rho_{f}\left(u_{w}\right)^{2}} ; & \mathrm{Nu}_{x}=-\frac{x q_{w}}{k_{f}\left(T_{f}-T_{\infty}\right)} ; \\
\tau_{w}=\mu_{f}\left(\frac{\partial u}{\partial z}\right)_{z=0} ; & q_{w}=-k_{f}\left(\frac{\partial T}{\partial z}\right)_{z=0} ;
\end{array}
$$

In Eq.(2.19), $\tau_{w}$ represents the wall shear stress at the shrinking surface and $q_{w}$ is the wall heat flux from the shrinking surface. Using the similarity transformation, the skin friction coefficient and local Nusselt number can be represented as

$$
C_{f} \sqrt{\operatorname{Re}_{x}}=\frac{f^{\prime \prime}(0)}{\sqrt{A}(1-\varphi)^{2.5}}, \quad \frac{\mathrm{Nu}_{x}}{\sqrt{\operatorname{Re}_{x}}}=-A^{-\frac{3}{2}} k \theta^{\prime}(0) .
$$

\section{Numerical solution}

The coupled nonlinear ODE in Eqs (2.15)-(2.16) are solved numerically through mathematical modeling in COMSOL Multiphysics. The following transformation has been used to reduce the above system of nonlinear equations into a set of second-order nonlinear ODE

$$
f=p, \quad \frac{d f}{d \xi}=q
$$

Equations (2.15)-(2.16) can now be written as 


$$
\begin{aligned}
& \frac{d p}{d \xi}=q, \\
& \left(1+\frac{3}{2} \varphi_{1} \lambda\right) \frac{d^{2} q}{d \xi^{2}}+(1-\varphi)^{2.5}\left[1-\varphi+\left(\frac{\rho_{s}}{\rho_{f}}\right) \varphi\right]\left[m p \frac{d q}{d \xi}-q^{2}\right]+ \\
& -\left[(1-\varphi)^{2.5} M+K_{p}\right] q-\mathrm{Gr} \theta=0, \\
& \frac{1}{\operatorname{Pr}}(k+R) \frac{d^{2} \theta}{d \xi^{2}}+\frac{\mathrm{Ec} m^{2}}{(1-\varphi)^{2.5}} q^{2}+\left[1-\varphi+\left\{\frac{\left(\rho C_{p}\right)_{s}}{\left(\rho C_{p}\right)_{f}}\right\} \varphi\right] m p \frac{d \theta}{d \xi}+\chi \theta=0, \\
& p=f_{w}, \quad q=A, \quad \frac{d \theta}{d \xi}=-B_{i}(1-\theta) \quad \text { at } \quad \xi=0, \\
& q \rightarrow 0, \quad \theta \rightarrow 0 \quad \text { as } \quad \xi \rightarrow \infty .
\end{aligned}
$$

Equations (3.2)-(3.4) with the help of Eq.(3.5) are solved using the finite element method through COMSOL under the coefficient form PDE option.

The above equations are converted into second-order nonlinear partial differential equations that are compatible for the mathematical modeling in COMSOL Multiphysics. Due to the similarity transformation, the above equations have been converted into one-dimensional form, therefore a line geometry has been used to define the region of the solution. Under the dimensionless category, the PDE tool has been used in the solution of the equations. During the solution process, Dirichlet boundary conditions are imposed to obtain the solution of the problem. The maximum element size of the mesh is taken as 0.004 and the maximum element growth rate is taken as 1.1 and resolutions of the narrow regions are taken as 1 . The table for the local skin friction coefficient and Nusselt number is as follows

Table 1. Value of the local skin friction coefficient and Nusselt number for different values of $M$ and $K_{p}$ at for $m=\mathrm{Gr}=\chi=2, \mathrm{Ec}=0.2, \operatorname{Pr}=0.71, f_{w}=0.1, B_{i}=0.1, k=R=0.5, A=-0.1$.

\begin{tabular}{|c|l|l|l|l|l|l|}
\hline $\boldsymbol{M}$ & \multicolumn{1}{|c|}{$\boldsymbol{0}$} & \multicolumn{1}{c|}{$\mathbf{0 . 2}$} & \multicolumn{1}{c|}{$\mathbf{0 . 5}$} & \multicolumn{1}{c|}{$\mathbf{1}$} & \multicolumn{1}{c|}{$\mathbf{2}$} & \multicolumn{1}{c|}{$\mathbf{3}$} \\
\hline$-f^{\prime \prime}(0)$ & 1.10132 & 1.34452 & 1.54432 & 1.98765 & 2.02456 & 2.14567 \\
\hline$-\theta^{\prime}(0)$ & 0.10564 & 0.10037 & 0.09987 & 0.095547 & 0.09324 & 0.09184 \\
\hline $\boldsymbol{K}_{\boldsymbol{p}}$ & $\mathbf{0 . 1}$ & $\mathbf{0 . 2}$ & $\mathbf{0 . 3}$ & $\mathbf{0 . 4}$ & $\mathbf{0 . 5}$ & $\mathbf{0 . 6}$ \\
\hline$-f^{\prime \prime}(0)$ & 1.11342 & 1.35435 & 1.56754 & 2.10345 & 2.11564 & 2.24565 \\
\hline$-\theta^{\prime}(0)$ & 0.09956 & 0.09656 & 0.09523 & 0.09503 & 0.09433 & 0.09368 \\
\hline
\end{tabular}

\section{Results and discussions}

The present results show the distributions of velocity and temperature for several values of dimensionless parameters. There are various physical parameters, which are involved in the differential equations. However, the results are obtained for different values of the important dimensionless parameters, which change the velocity and temperature distribution significantly.

Figure 2 indicates the velocity distribution for numerous values of $A$. The flow velocity increases by increasing the value of the shrinking parameter $(A)$. Increasing the shrinking parameter enhances the velocity 
distribution leading to a thicker momentum boundary layer. The velocity distribution for the permeability parameter is presented in Fig.3. The velocity of nanofluid decreases by increasing the values of the permeability parameter. It has been observed from the result that porous permeability in the fluid exerts a resistant force to reduce the velocity distribution. This force reduces the momentum boundary layer in the fluid.

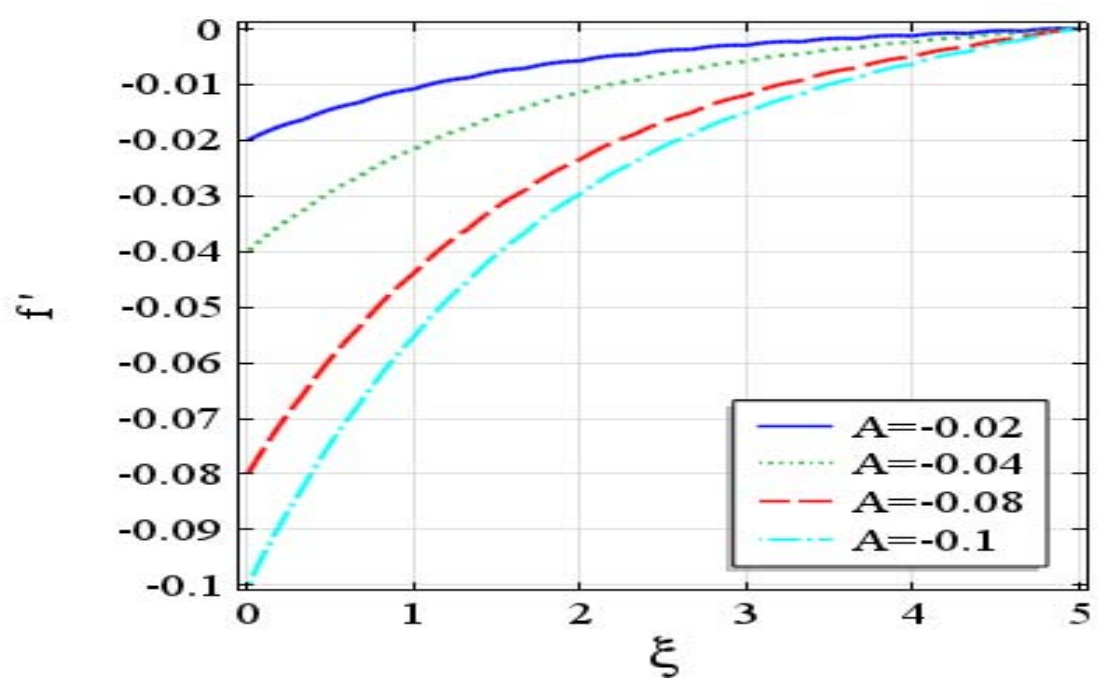

Fig.2. Velocity distribution for $m=\mathrm{Gr}=\chi=2, \quad M=K_{p}=\mathrm{Ec}=0.2, \operatorname{Pr}=0.71, \quad f_{w}=0.1, \quad B_{i}=0.1$, $k=R=0.5$.

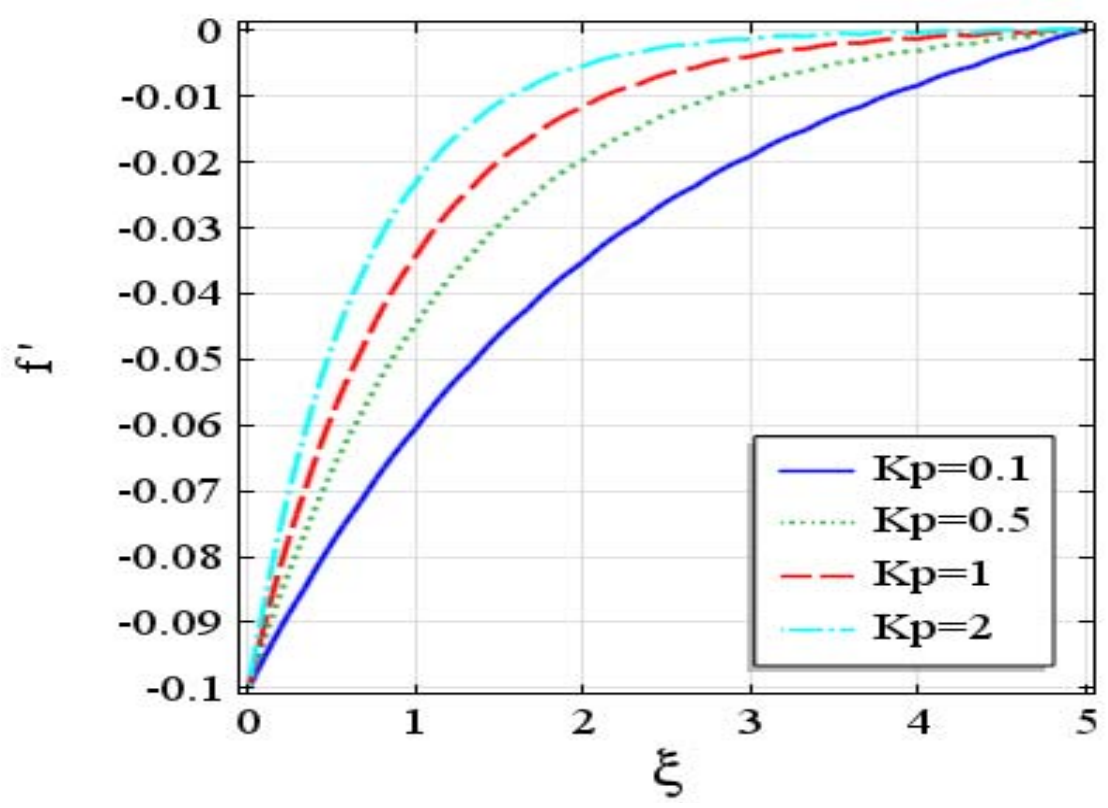

Fig.3. Velocity distribution for $m=\mathrm{Gr}=\chi=2, M=\mathrm{Ec}=0.2, \operatorname{Pr}=0.71, f_{w}=0.1, B_{i}=0.1, k=R=0.5$, $A=-0.1$.

Figure 4 depicts the velocity distribution for various values of magnetic numbers. The velocity of the fluid decreases for increasing the value of the magnetic parameter. The result indicates that the magnetic force acting on the fluid creates an additional resistance on the fluid which generates the rotational viscosity in the fluid. Increasing the viscosity of the fluid, the velocity distribution decreases. The fluid reaches a steady-state region quickly in the presence of the magnetic number. The fluid is conducting and the magnetic field is directed in the transverse direction. Therefore, due to the Lorentz force, the fluid velocity decreases 
for increasing the values of the magnetic number. The effect of the volume fraction of nanoparticles on the velocity distribution is presented in Fig.5. For increasing values of the volume fraction the velocity distributions increase since the volume fraction empowers the fluid motion. Figure 6 represents the velocity distribution in the absence of thermal and heat absorption effects. Without heat and thermal effects, the pattern of velocity distribution is similar as published in the results of Nayak [2].

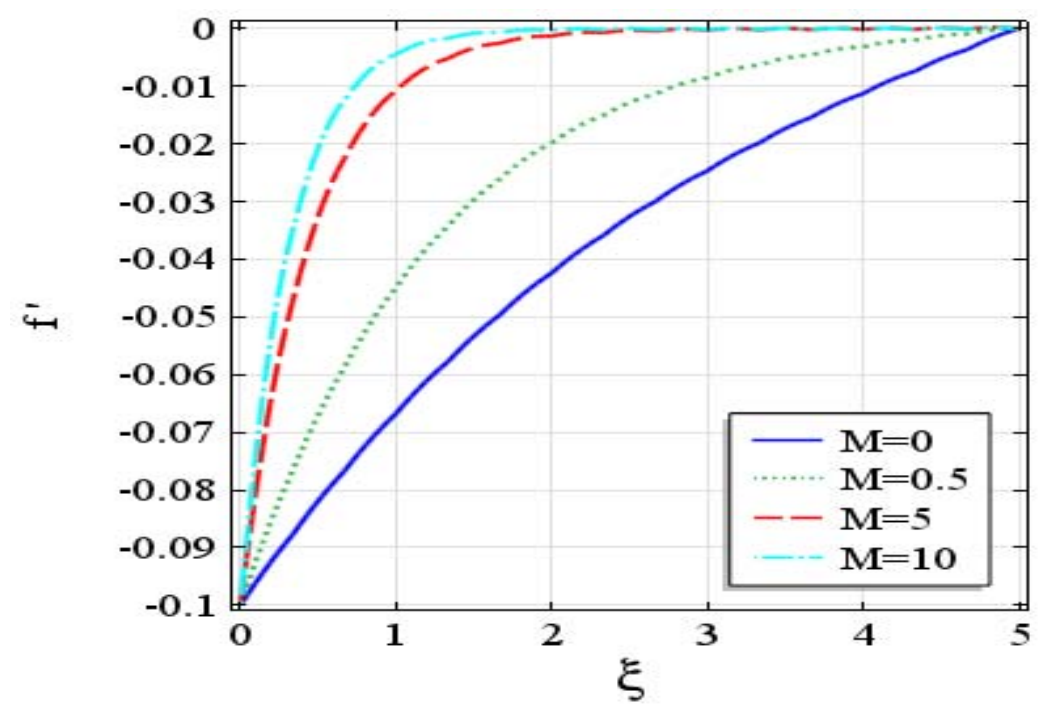

Fig.4. Velocity distribution for $m=\mathrm{Gr}=\chi=2, \quad K_{p}=\mathrm{Ec}=0.2, \quad \operatorname{Pr}=0.71, \quad f_{w}=0.1, \quad B_{i}=0.1$, $k=R=0.5, A=-0.1$.

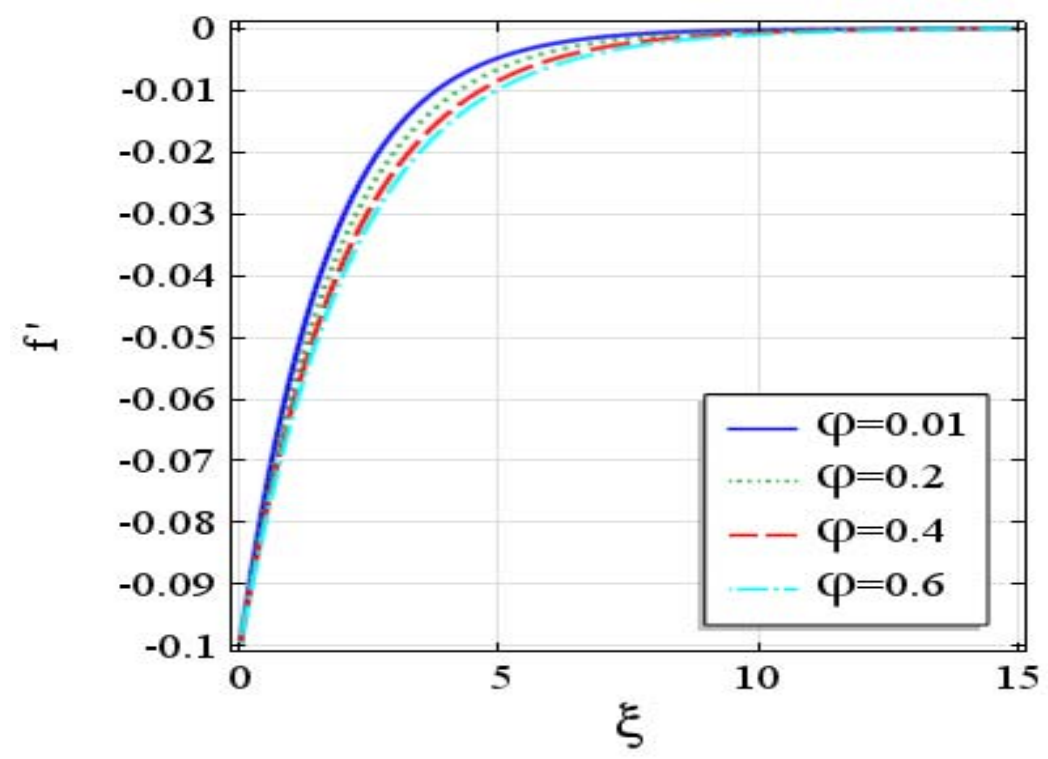

Fig.5. Velocity distribution for $m=\mathrm{Gr}=\chi=2, \quad M=\mathrm{Ec}=K_{p}=0.2, \operatorname{Pr}=0.71, \quad f_{w}=0.1, \quad B_{i}=0.1$, $k=R=0.5, A=-0.1$. 


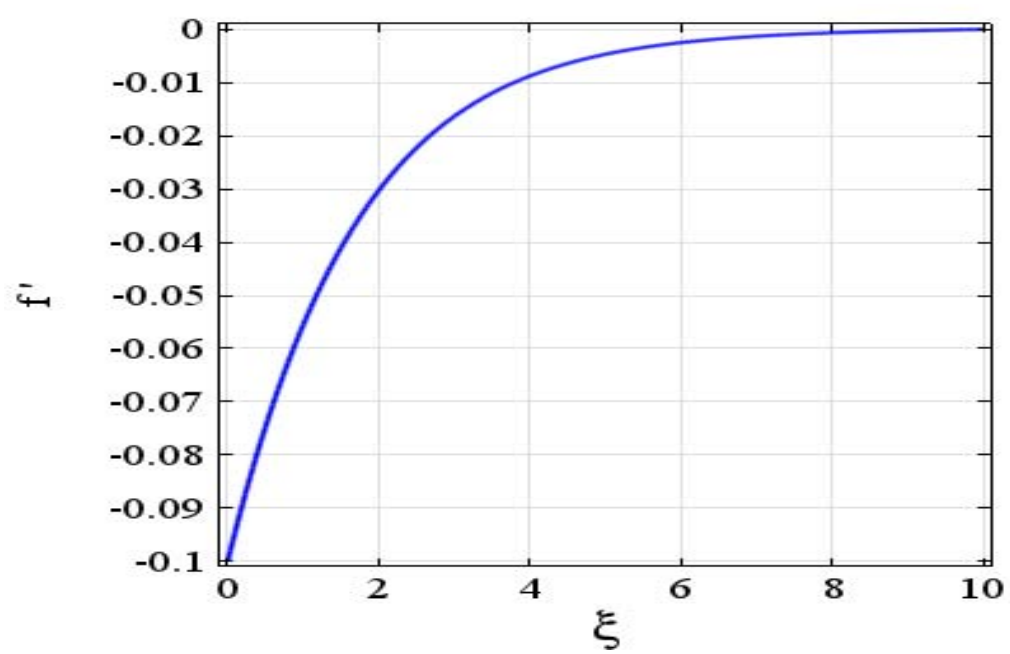

Fig.6. Velocity distribution without thermal and heat absorption parameter for $m=2, M=\mathrm{Ec}=K_{p}=0.2$,

$\operatorname{Pr}=0.71, f_{w}=0.1, B_{i}=0.1, k=R=0.5, A=-0.1$.

Figure 7 represents the heat transfer for different values of the shrinking parameter. Increasing the values of the shrinking parameter increases the heat transfer in the nanofluid. The shrinking parameter keeps the nanoparticles closer to each other. It increases the temperature of the fluid. Figure 8 depicts the temperature distribution for different values of $B_{i}$. Increasing the values of $B_{i}$ increases the heat transfer in the nanofluid. increasing the values of the Biot number increases the thickness of the thermal boundary layer.

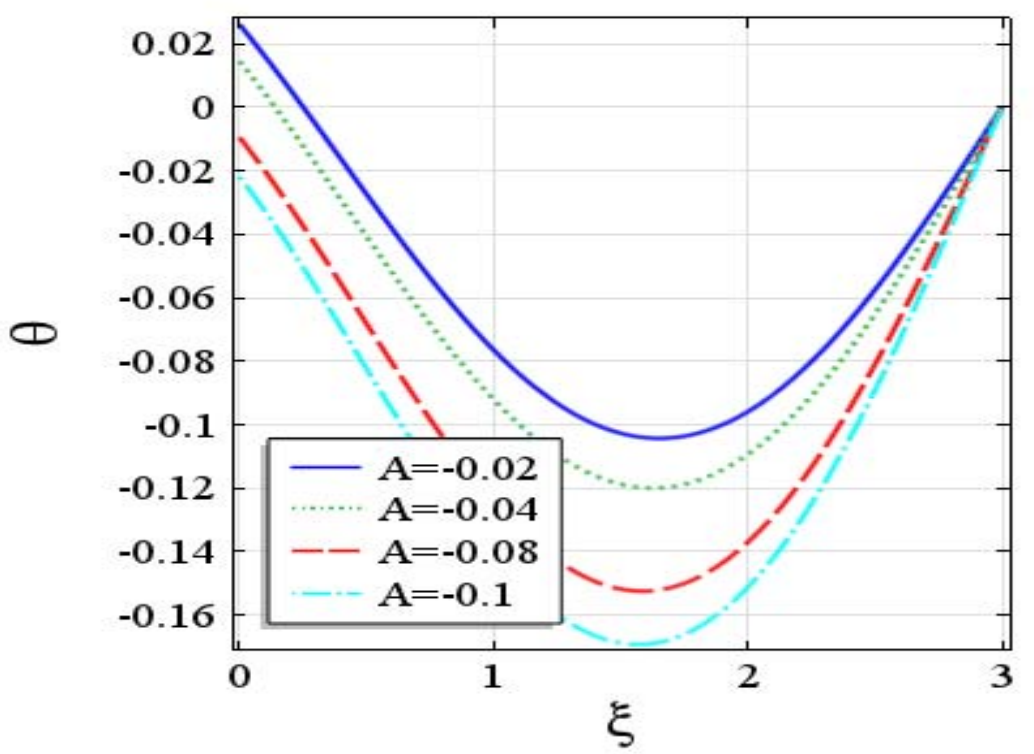

Fig.7. Temperature distribution for $m=\mathrm{Gr}=\chi=2, M=K_{p}=\mathrm{Ec}=0.2, \operatorname{Pr}=0.71, f_{w}=0.1, \quad B_{i}=0.1$, $k=R=0.5$. 


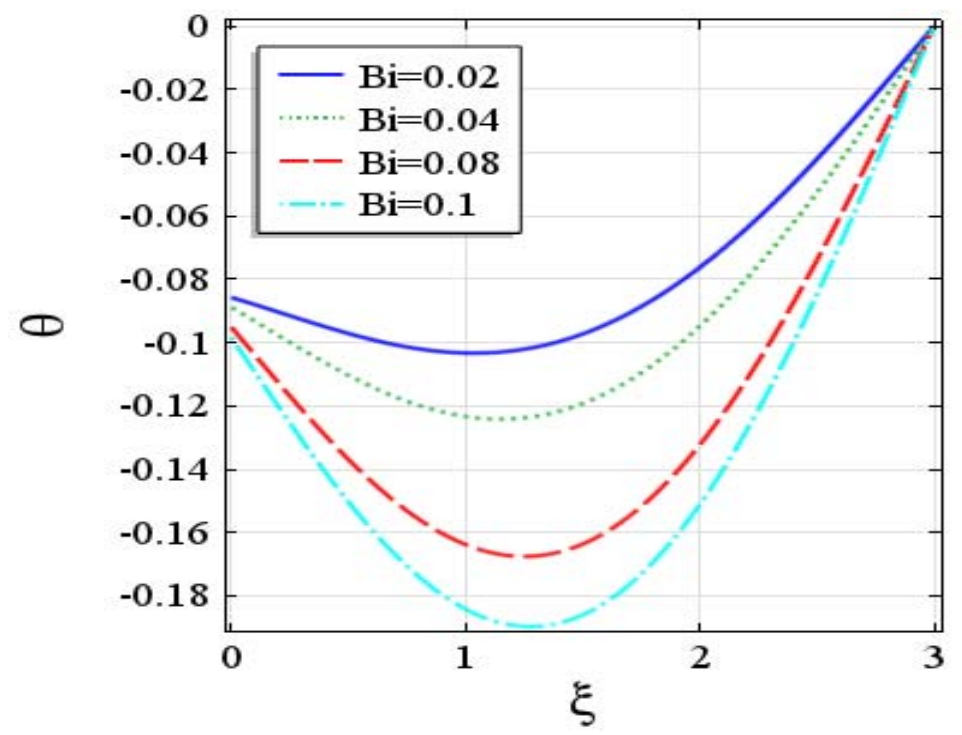

Fig.8. Temperature distribution for $m=\mathrm{Gr}=\chi=2, M=\mathrm{Ec}=K_{p}=0.2, \operatorname{Pr}=0.71, f_{w}=0.1, k=R=0.5$, $A=-0.1$.

Figure 9 shows the temperature distributions in the presence of the heat absorption parameter. At $\chi=1$, the temperature distribution in the nanofluid is maximum as compared to other values of heat absorption coefficients. Figure 10 shows the distribution of temperature for different values of the thermal Grashof number (Gr). It increases the thermal boundary layer in the fluid. Heat transfer in the nanofluid increases by increasing the thermal Grashof number. Moreover, an increase in volume fraction values also increases the fluid heat transfer, as shown in Fig.11. Increasing the volume fraction of the nanofluid, the Lorentz force will be more effective in shrinking the momentum boundary layer thickness. However, it will increase the temperature distribution in the fluid since magnetization is a function of temperature.

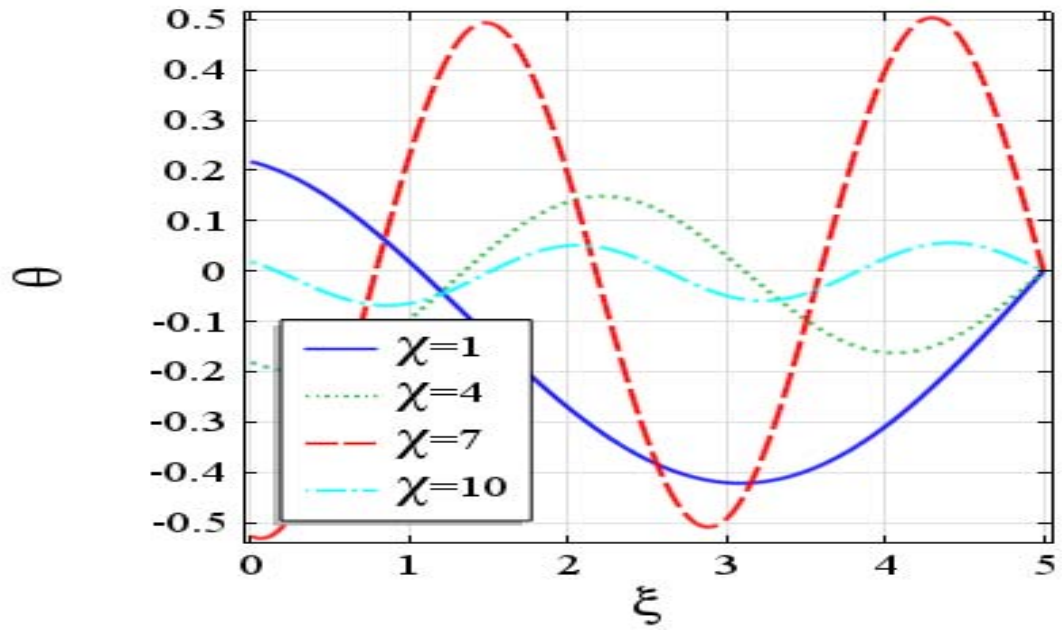

Fig.9. Temperature distribution for $m=\mathrm{Gr}=2, \quad M=\mathrm{Ec}=K_{p}=0.2, \operatorname{Pr}=0.71, f_{w}=0.1, \quad B_{i}=0.1$, $k=R=0.5, A=-0.1$. 


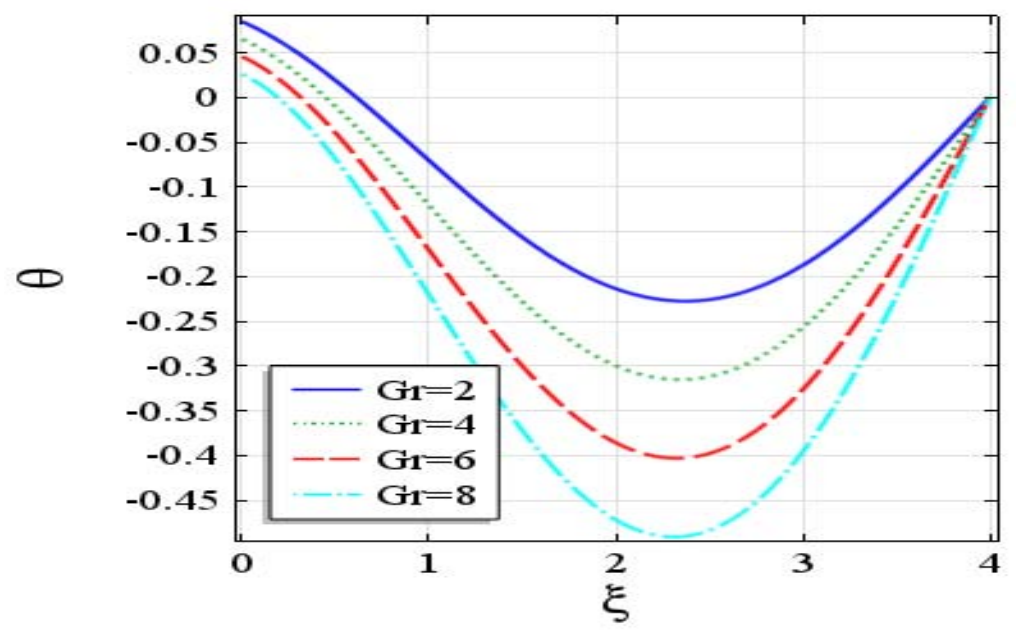

Fig.10. Temperature distribution for $m=\chi=2, \quad M=\mathrm{Ec}=K_{p}=0.2, \quad \operatorname{Pr}=0.71, \quad f_{w}=0.1, \quad B_{i}=0.1$, $k=R=0.5, A=-0.1$.

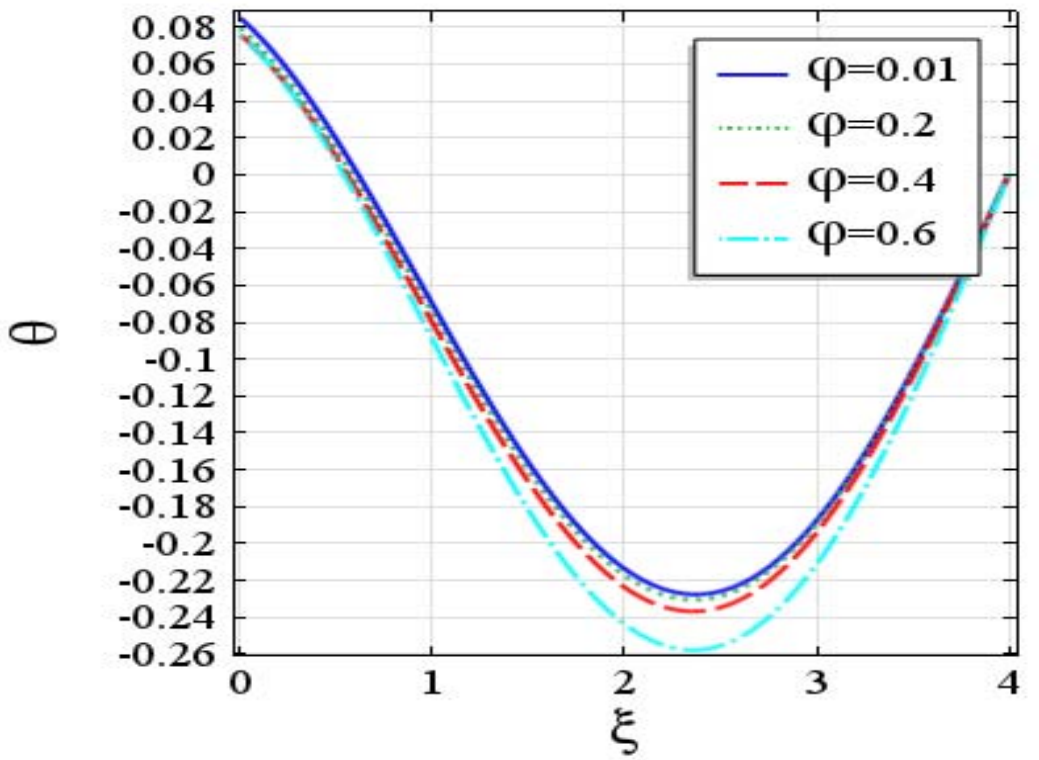

Fig.11. Temperature distribution for $m=\mathrm{Gr}=\chi=2, M=\mathrm{Ec}=K_{p}=0.2, \operatorname{Pr}=0.71, f_{w}=0.1, B_{i}=0.1$, $k=R=0.5, A=-0.1$.

The temperature and the permeability parameter $K_{p}$ are inversely proportional to each other in the entire flow region. It has been observed in the current study that the porous permeability parameter reduces the heat transfer as shown in Fig.12. In the presence of the porous matrix, there are small decreases in the heat transfer distribution. However, far from the porous plate, there is no impact of the permeability parameter in the temperature distributions. This fact reveals that increasing values of $M$ enhance the fluid temperature near the boundary layer, however, far from the boundary layer, the heat transfer decreases as presented in Fig.13. Temperature and magnetization are proportional to each other. Therefore, increasing the magnetic field intensity increases the heat transfer in the fluid. The temperature distribution increases for 
increasing the radiation parameter and it does not make any dramatic change in the growth of the thermal boundary layer.

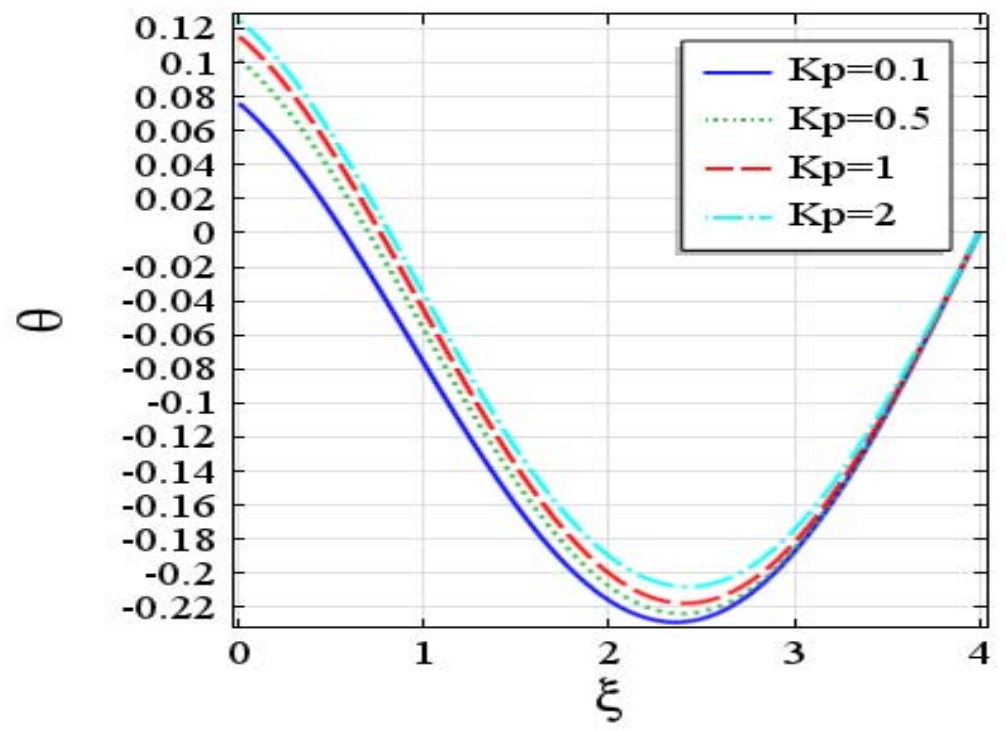

Fig.12. Temperature distribution for $m=\mathrm{Gr}=\chi=2, \quad M=\mathrm{Ec}=0.2, \operatorname{Pr}=0.71, \quad f_{w}=0.1, \quad B_{i}=0.1$, $k=R=0.5, A=-0.1$.

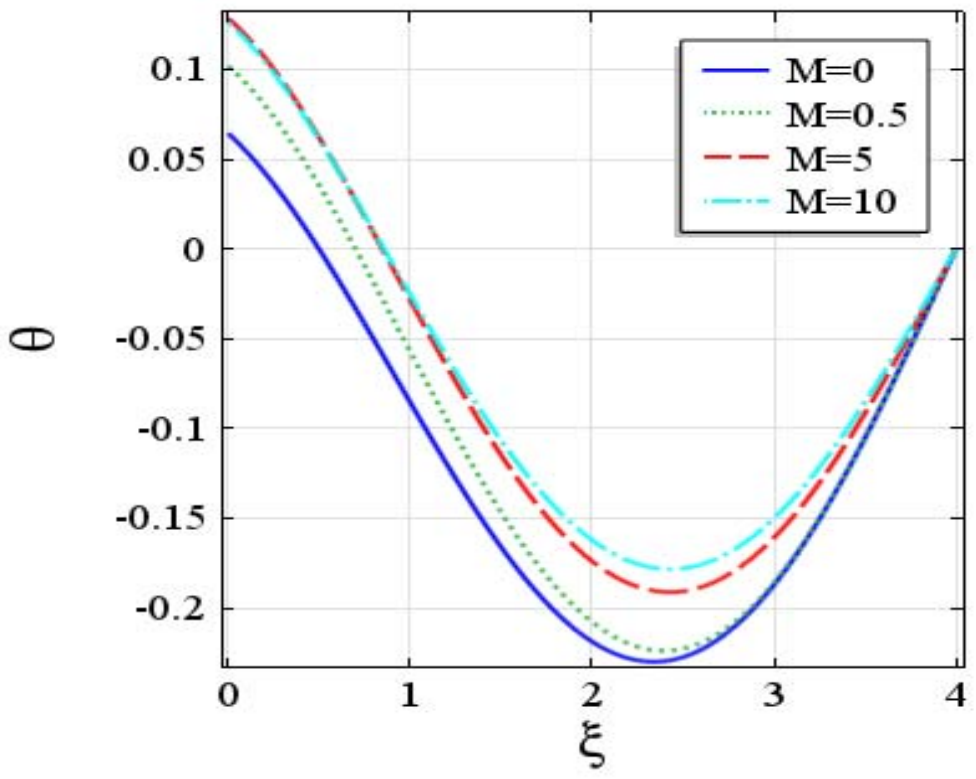

Fig.13. Temperature distribution for $m=\mathrm{Gr}=\chi=2, \mathrm{Ec}=K_{p}=0.2, \operatorname{Pr}=0.71, f_{w}=0.1, \quad B_{i}=0.1$, $k=R=0.5, A=-0.1$.

For a cooling system, the radiation parameter should be very small. Figure 15 shows the temperature distribution without thermal expression $g \beta\left(T-T_{\infty}\right)$ and heat absorption expression $\frac{Q_{0}}{\left(\rho C_{p}\right)_{n}}\left(T-T_{\infty}\right)$. In this case, the heat transfer pattern remains similar to the pattern previously published in [2]. Table 1 represents the values of the local skin friction coefficient and the local Nusselt number. It has been observed 
from the results that the local skin friction gets enhanced for increasing the values of the magnetic number and permeability parameter. However, an increase in the values of the magnetic number and medium permeability decreases the values of the Nusselt number.

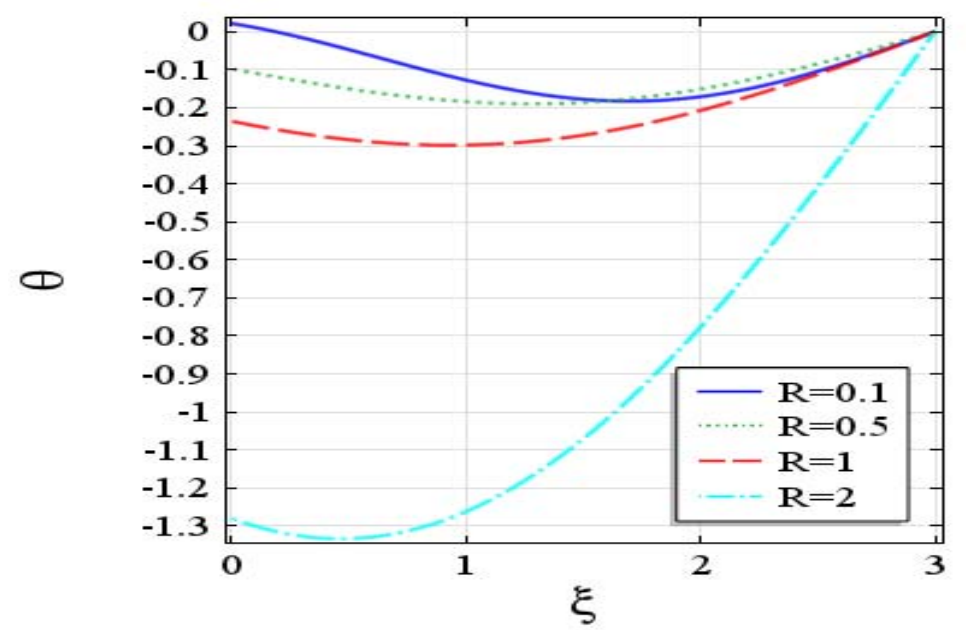

Fig.14. Temperature distribution for $m=\mathrm{Gr}=\chi=2, M=\mathrm{Ec}=K_{p}=0.2, \operatorname{Pr}=0.71, f_{w}=0.1, B_{i}=0.1$, $k=0.5, A=-0.1$.

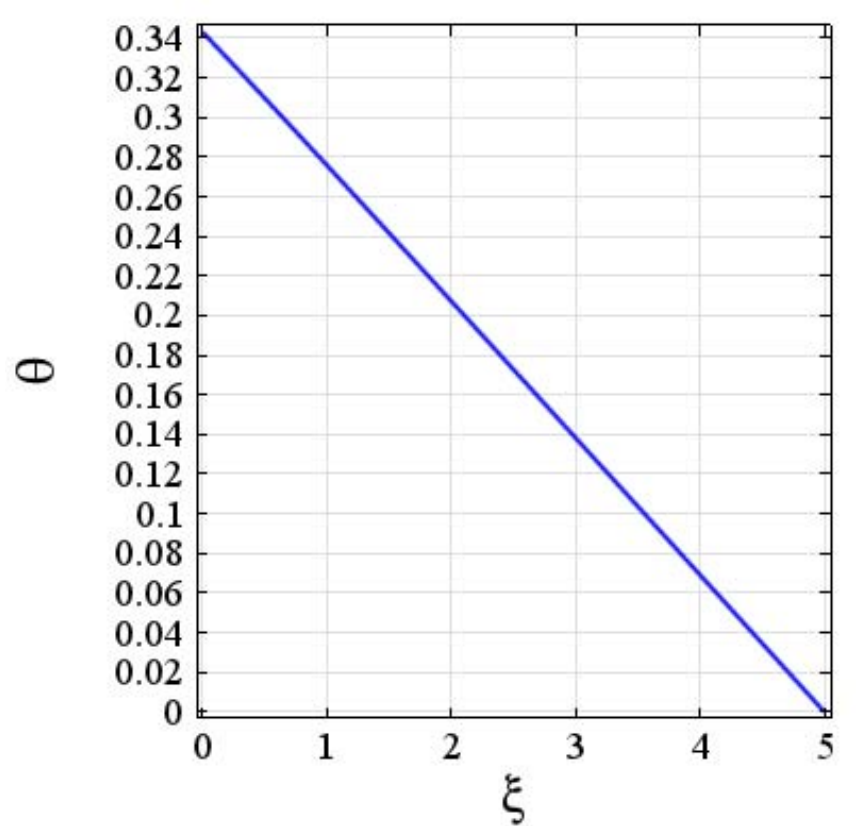

Fig.15. Temperature distribution without thermal and heat absorption parameter for $m=2$, $M=\mathrm{Ec}=K_{p}=0.2, \operatorname{Pr}=0.71, f_{w}=0.1, k=R=0.5, A=-0.1$.

\section{Conclusions}

The conclusions from the current study can be summarized as follows:

1. The presence of the magnetic parameter $(M)$, the permeability parameter $\left(K_{p}\right)$ impedes the nanofluid motion. However, the fluid velocity has increased with increasing the values of the shrinking parameter $(A)$ and the volume fraction of nanoparticle $(\varphi)$ whereas other parameters have not shown any significant impact on the fluid velocity. 
2. Heat transfer in the thermal boundary increases for increasing values of the shrinking parameter $(A)$, Biot number $\left(\mathrm{B}_{\mathrm{i}}\right)$, thermal Grashof number $(\mathrm{Gr})$, nanoparticle volume fraction $(\varphi)$ and radiation parameter $(R)$. It has been observed that heat transfer has been decreased due to the presence of heat absorption and permeability factors in the thermal boundary layer. In the presence of the magnetic parameter, variations in the heat transfer are observed with the distance from the thermal boundary layer.

\section{Nomenclature}

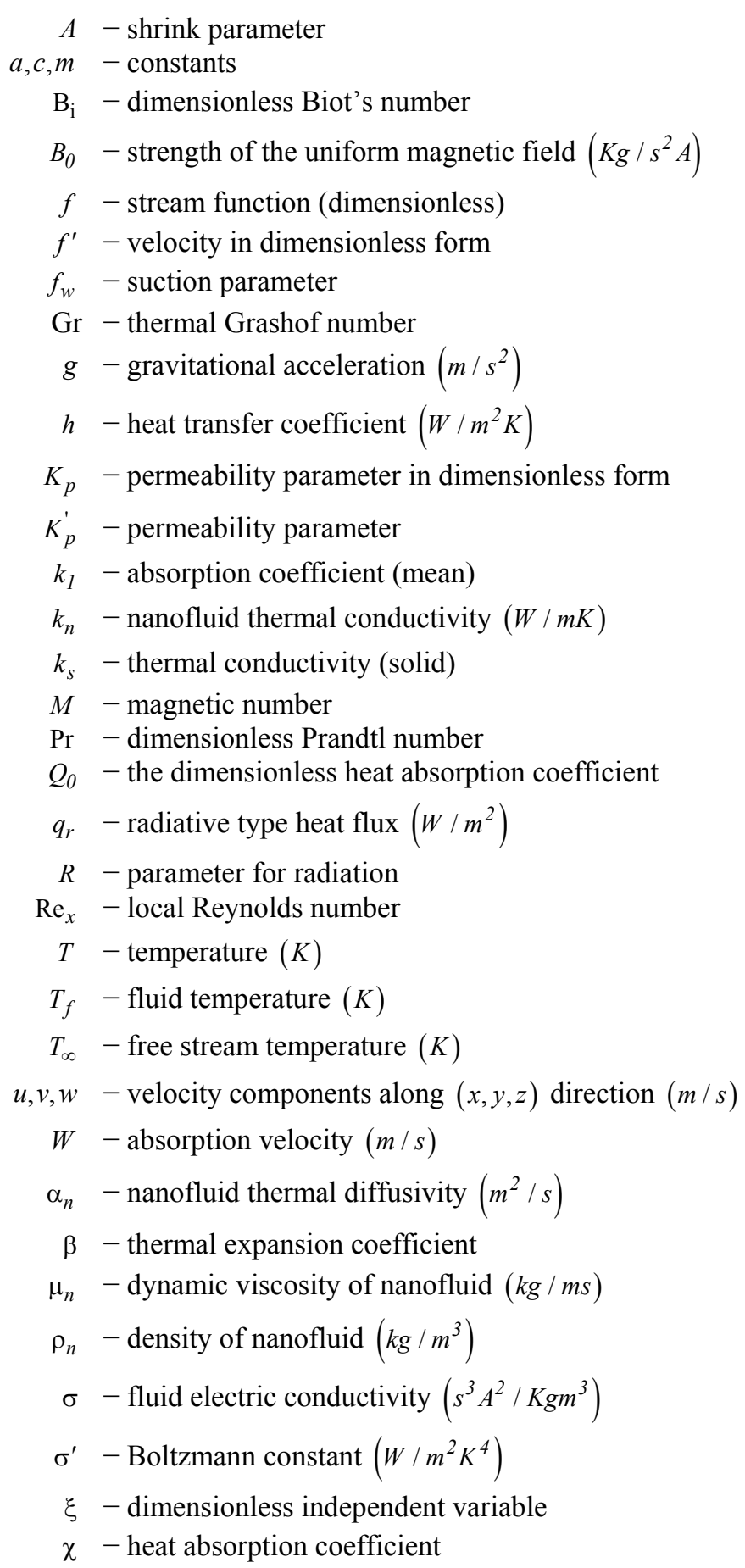




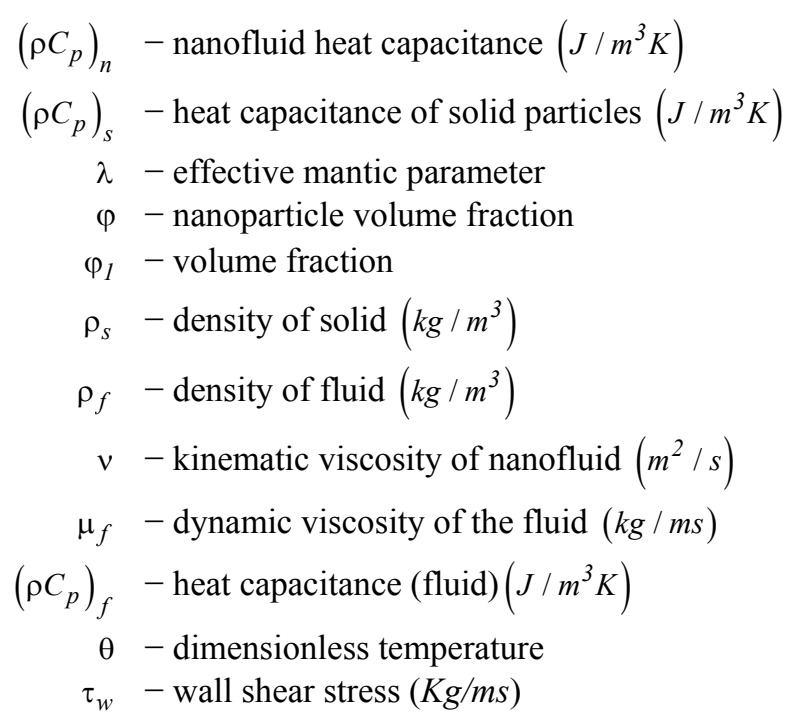

\section{References}

[1] Mahalakshmi, Thangavelu, Nagarajan Nithyadevi, Hakan F. Oztop and Nidal Abu-Hamdeh (2018): MHD mixed convective heat transfer in a lid-driven enclosure filled with Ag-water nanofluid with center heater.International Journal of Mechanical Sciences, vol.142, pp.407-419.

[2] Nayak M.K. (2017): MHD 3D flow and heat transfer analysis of nanofluid by shrinking surface inspired by thermal radiation and viscous dissipation. - International Journal of Mechanical Sciences, vol.124, pp.185-193.

[3] Hayat, Tasawar, Maria Imtiaz and Ahmed Alsaedi (2015): MHD 3D flow of nanofluid in presence of convective conditions. - Journal of Molecular Liquids, vol.212, pp.203-208.

[4] Elazem, Nader Y. Abd and Abdelhalim Ebaid (2017): Effects of partial slip boundary condition and radiation on the heat and mass transfer of MHD-nanofluid flow. - Indian Journal of Physics, 91.12, pp.1599-1608.

[5] Bhargava, Rama and Mania Goyal (2015): An efficient hybrid approach for simulating MHD nanofluid flow over a permeable stretching sheet. - Mathematical Analysis and its Applications, Springer, New Delhi, pp.701-714.

[6] Tamim, Hossein, Saeed Dinarvand, Reza Hosseini and Pop I. (2014): MHD mixed convection stagnation-point flow of a nanofluid over a vertical permeable surface: a comprehensive report of dual solutions. - Heat and Mass Transfer, 50.5, pp.639-650.

[7] Hayat T., Rashid M., Imtiaz M. and Alsaedi A. (2017): MHD effects on a thermo-solutal stratified nanofluid flow on an exponentially radiating stretching sheet. - Journal of Applied Mechanics and Technical Physics, 58.2, pp.214-223.

[8] Kumar R., Raju C.S.K., Sekhar K.R. and Reddy G.V. (2017): Three dimensional MHD ferrous nanofluid flow over a sheet of variable thickness in slip flow regime. - Journal of Mechanics, pp.1-12.

[9] Vajravelu K. and Prasad K.V. (2012): Heat transfer phenomena in a moving nanofluid over a horizontal surface. Journal of Mechanics, 28.3, pp.579-588.

[10] Biswas, Rajib and Sarder Firoz Ahmmed (2018): Effects of hall current and chemical reaction on magnetohydrodynamics unsteady heat and mass transfer of Casson nanofluid flow through a vertical plate. Journal of Heat Transfer, 140.9, 092402.

[11] Hayat, Tasawar, Arsalan Aziz, Taseer Muhammad, and Ahmed Alsaedi. (2017): Three-dimensional flow of nanofluid with heat and mass flux boundary conditions. - Chinese Journal of Physics, 55.4, pp.1495-1510.

[12] Sudhagar, Palani, Peri K. Kameswaran and B. Rushi Kumar (2017): Magnetohydrodynamics mixed convection flow of a nanofluid in an isothermal vertical cone. - Journal of Heat Transfer, 139.3.

[13] Das K., Pinaki Ranjan Duari and Kundu P.K. (2016): Effects of magnetic field on an unsteady mixed convection flow of nanofluids containing spherical and cylindrical nanoparticles. - Journal of Heat Transfer, 138.6, 061901. 
[14] Nandkeolyar, Raj, Peri K. Kameswaran, Sachin Shaw and Precious Sibanda (2014): Heat transfer on nanofluid flow with homogeneous-heterogeneous reactions and internal heat generation. - Journal of Heat Transfer, $136.12,122001$.

[15] Nandkeolyar R., Motsa S.S. and Sibanda P. (2013): Viscous and Joule heating in the stagnation point nanofluid flow through a stretching sheet with homogenous-heterogeneous reactions and nonlinear convection. - Journal of Nanotechnology in Engineering and Medicine, 4.4, 041002.

[16] Mustafa M., S. Hina, T. Hayat and A. Alsaedi (2013): Slip effects on the peristaltic motion of nanofluid in a channel with wall properties. - Journal of Heat Transfer, 135.4, 041701.

[17] Hayat T., Numra Gull M. Farooq and B. Ahmad (2015): Thermal radiation effect in MHD flow of Powell-Eyring nanofluid induced by a stretching cylinder. - Journal of Aerospace Engineering, 29.1, 04015011.

[18] Hayat, Tasawar, Taseer Muhammad, Sabir Ali Shehzad and Ahmed Alsaedi. (2015): Three-dimensional flow of Jeffrey nanofluid with a new mass flux condition. - Journal of Aerospace Engineering, 29.2, 04015054.

[19] Ramzan M., Bilal M., Jae Dong Chung, Dian Chen Lu and Farooq U. (2017): Impact of generalized Fourier's and Fick's laws on MHD 3D second grade nanofluid flow with variable thermal conductivity and convective heat and mass conditions. - Physics of Fluids, 29.9, 093102.

[20] Chamkha A.J., Rashad A.M., Mansour M.A., Armaghani T. and Ghalambaz M. (2017): Effects of heat sink and source and entropy generation on MHD mixed convection of a Cu-water nanofluid in a lid-driven square porous enclosure with partial slip. - Physics of Fluids, 29.5, 052001.

[21] Mohebbi, Rasul, Mohsen Izadi and Chamkha Ali J. (2017): Heat source location and natural convection in a Cshaped enclosure saturated by a nanofluid. - Physics of Fluids, 29.12, 122009.

[22] Reddy, B. Siva Kumar, M. Veera Krishna, KV Surya Narayana Rao, and R. Bhuvana Vijaya. (2018): HAM Solutions on MHD flow of nano-fluid through saturated porous medium with Hall effects. - Materials Today: Proceedings, 5.1, pp.120-131.

[23] Ibrahim, Wubshet (2017): Magnetohydrodynamic (MHD) boundary layer stagnation point flow and heat transfer of a nanofluid past a stretching sheet with melting. - Propulsion and Power Research, 6.3, pp.214-222.

[24] Mahalakshmi, Thangavelu, Nagarajan Nithyadevi, Hakan F. Oztop, and Nidal Abu-Hamdeh. (2018): MHD mixed convective heat transfer in a lid-driven enclosure filled with Ag-water nanofluid with center heater.International Journal of Mechanical Sciences, vol.142, pp.407-419.

[25] Ramzan, Muhammad, Hina Gul and Seifedine Kadry (2019): Onset of Cattaneo-Christov heat flux and thermal stratification in ethylene-glycol based nanofluid flow containing carbon nanotubes in a rotating frame. - IEEE Access, vol.7, pp.146190-146197.

[26] Khan, Umair, Shafiq Ahmad, Muhammad Ramzan, Muhammad Suleman, Dianchen Lu and Saba Inam. (2019): Numerical simulation of Darcy-Forchheimer 3D unsteady nanofluid flow comprising carbon nanotubes with Cattaneo-Christov heat flux and velocity and thermal slip conditions. - Processes, 7.10, 687.

[27] Li, Zhixiong, Ahmad Shafee, R. Kandasamy, M. Ramzan, and Qasem M. Al-Mdallal (2019): Nanoparticle transportation through a permeable duct with Joule heating influence. - Microsystem Technologies, 25.9, pp.3571-3580.

[28] Ramzan, Muhammad, Mutaz Mohammad and Fares Howari (2019): Magnetized suspended carbon nanotubes based nanofluid flow with bio-convection and entropy generation past a vertical cone. - Scientificreports, 9.1, pp.1-15.

[29] Ramzan, Muhammad, Mutaz Mohammad, Fares Howari, and Jae Dong Chung. (2019): Entropy analysis of carbon nanotubes based nanofluid flow past a vertical cone with thermal radiation. - Entropy, 21.7, 642.

[30] Alebraheem, Jawdat and Ramzan M. (2019): Flow of nanofluid with Cattaneo-Christov heat flux model. Applied Nanoscience, pp.1-11.

[31] Farooq U., Lu D.C., Munir S., Suleman M. and Ramzan M. (2019): Flow of rheological nanofluid over a static wedge. - Journal of Nanofluids, 8.6, pp.1362-1366.

[32] Li, Zhixiong, M. Ramzan, Ahmad Shafee, S. Saleem, Qasem M. Al-Mdallal and Ali J. Chamkha. (2019): 
Numerical approach for nanofluid transportation due to electric force in a porous enclosure. - Microsystem Technologies, 25.6, pp.2501-2514.

[33] Bilal M. and Ramzan M. (2019): Hall current effect on unsteady rotational flow of carbon nanotubes with dust particles and nonlinear thermal radiation in Darcy-Forchheimer porous media. - Journal of Thermal Analysis and Calorimetry, vol.138, pp.3127-3137.

[34] Farooq, Umer, Dianchen Lu, Shahzad Munir, Muhammad Ramzan, Muhammad Suleman and Shahid Hussain (2019): MHD flow of Maxwell fluid with nanomaterials due to an exponentially stretching surface. - Scientific Reports 9.1, 7312.

[35] Lu, Dianchen, Muhammad Ramzan, Mutaz Mohammad, Fares Howari and Jae Dong Chung. (2019): A thin film flow of nanofluid comprising carbon nanotubes influenced by Cattaneo-Christov heat flux and entropy generation. - Coatings, 9.5, 296.

[36] Suleman, Muhammad, Muhammad Ramzan, Shafiq Ahmad, and Dianchen Lu. (2019): Numerical simulation for homogeneous-heterogeneous reactions and Newtonian heating in the silver-water nanofluid flow past a nonlinear stretched cylinder. - Physica Scripta, 94.8, 085702.

[37] Lu, Dianchen, Zhixiong Li, M. Ramzan, Ahmad Shafee, and Jae Dong Chung. (2019): Unsteady squeezing carbon nanotubes based nano-liquid flow with Cattaneo-Christov heat flux and homogeneous-heterogeneous reactions. - Applied Nanoscience, 9.2, pp.169-178.

[38] Suleman, Muhammad, Muhammad Ramzan, Shafiq Ahmad, Dianchen Lu, Taseer Muhammad and Jae Dong Chung (2019): A numerical simulation of silver-water nanofluid flow with impacts of Newtonian heating and homogeneous-heterogeneous reactions past a nonlinear stretched cylinder. - Symmetry, 11.2, 295.

[39] Ramzan, Muhammad, Mohsen Sheikholeslami, Maria Saeed and Jae Dong Chung (2019): On the convective heat and zero nanoparticle mass flux conditions in the flow of $3 D$ MHD couple stress nanofluid over an exponentially stretched surface. - Scientific Reports 9.1, 562.

[40] Li, Zhixiong, Sheikholeslami M., Ahmad Shafee, Ramzan M., Kandasamy R. and Qasem M. Al-Mdallal (2019): Influence of adding nanoparticles on solidification in a heat storage system considering radiation effect. Journal of Molecular Liquids, vol.273, pp.589-605.

[41] Sheikholeslami M., Ahmad Shafee, M. Ramzan and Zhixiong Li (2018): Investigation of Lorentz forces and radiation impacts on nanofluid treatment in a porous semi annulus via Darcy law.- Journal of MolecularLiquids, vol.272, pp.8-14.

[42] Suleman, Muhammad, Muhammad Ramzan, Madiha Zulfiqar, Muhammad Bilal, Ahmad Shafee, Jae Dong Chung, Dianchen Lu and Umer Farooq (2018): Entropy analysis of 3D non-Newtonian MHD nanofluid flow with nonlinear thermal radiation past over exponential stretched surface. - Entropy, 20.12, 930.

[43] Hsiao and Kai-Long (2017): To promote radiation electrical MHD activation energy thermal extrusion manufacturing system efficiency by using Carreau-nanofluid with parameters control method. - Energy, vol.130, pp.486-499.

[44] Hsiao and Kai-Long (2017): Combined electrical MHD heat transfer thermal extrusion system using Maxwell fluid with radiative and viscous dissipation effects. - Applied Thermal Engineering, vol.112, pp.1281-1288.

[45] Hsiao and Kai-Long (2017): Micropolar nanofluid flow with MHD and viscous dissipation effects towards a stretching sheet with multimedia feature. - International Journal of Heat and Mass Transfer, vol.112, pp.983-990.

[46] Hsiao and Kai-Long (2016): Stagnation electrical MHD nanofluid mixed convection with slip boundary on a stretching sheet. - Applied Thermal Engineering, vol.98, pp.850-861. 